\title{
MÉTHODE DES MICROHABITATS : VALIDATION ET PERSPECTIVES.
}

\author{
M. POUILLY, Y. SOUCHON
}

CEMAGREF, Division Biologie des Ecosytèmes Aquatiques, Laboratoire d'Hydroécologie Quantitative, 3 bis Quai Chauveau, CP 220, 69336 Lyon Cedex 09, France.

\section{RÉSUMÉ}

La méthode des microhabitats (Instream Flow Incremental Methodology, en anglais) est l'outil actuellement le plus utilisé pour la simulation des capacités d'accueil des rivières. Elle consiste à coupler des informations obtenues à partir d'un modèle hydraulique et d'un modèle biologique. Cet outil peut s'avérer utile notamment dans les négociations qui visent à définir un débit à réserver à l'aval des ouvrages dérivant l'eau.

De nombreuses interrogations subsistent néanmoins quant à sa signification biologique. Les hypothèses sous-jacentes ainsi que les conditions expérimentales à réunir pour valider la démarche sont présentées et discutées.

Les critiques afférentes à chacun de ces modèles permettent de présenter les principaux développements actuels et les axes de recherche à promouvoir pour améliorer l'ensemble de la démarche.

Mots-clés : méthode des microhabitats, préférences d'habitat des poissons, capacité d'accueil, modèles biologiques.

\section{MICROHABITAT METHODOLOGY : VALIDATION AND PERSPECTIVES.}

\section{ABSTRACT}

Instream Flow Incremental Methodology ("microhabitat" methodology, in French) is presently the most used tool to evaluate stream carrying capacity by crossing informations from biological and hydraulic models. It is used in negotiation for minimum instream flow determination in regulated rivers.

However, the biological validity of the methodology remains its main uncertainty. Hypotheses and experimental conditions for validation are presented and discussed.

The discussion of each model explains its present development and future research necessary to improve the methodology.

Key-words : IFIM procedure, fish habitat use, carrying capacity, biological models.

\section{INTRODUCTION}

Confrontés à la nécessité d'établir des classements des cours d'eau selon leur valeur piscicole, les gestionnaires des pêches ont depuis longtemps tenté de sélectionner les critères qui les discriminaient le mieux. De très nombreuses méthodes ont vu le jour (revue de FAUSCH et al., 1988). Elles tentent de prédire la biomasse de poissons (salmonidés en général), en fonction de diverses métriques parmi lesquelles celles décrivant l'habitat occupent une place de choix. Le poids respectif de ces métriques varie selon les régions (FAUSCH et al., 1988), si bien que les possibilités de généralisation des modèles sont souvent faibles. De plus, la plupart de ces modèles ne prennent pas en compte la dynamique des phénomènes, ce qui les rend difficilement opérants en situation de prévision, lorsque les conditions physiques d'un cours d'eau sont modifiées. 
De nouvelles approches ont été proposées au début des années 1980 pour aider à mieux définir les conditions d'utilisation de l'eau par différents usages concurrentiels, tout en maintenant un débit favorable au développement de la vie aquatique. L'enjeu est donc tout autre, puisqu'il s'agit de prévoir l'évolution d'une rivière et de sa faune en fonction du débit.

Les premières méthodes essentiellement physiques basées sur l'hydrologie ou l'hydraulique ont rapidement cédé la place à une méthodologie plus élaborée, connue en français sous le nom de méthode des microhabitats (SOUCHON et al., 1989 ; SABATON et MIQUEL, 1993), originaire des U.S.A. (IFIM, "Instream Flow Incremental Methodology" : BOVEE et MILHOUS, 1978 ; BOVEE, 1982). Elle couple un modèle hydraulique simulant les conditions hydrodynamiques d'un secteur de cours d'eau pour plusieurs débits et des modèles biologiques représentant les préférences d'habitat des poissons pour différentes conditions hydrodynamiques. Elle permet ainsi de prédire la quantité d'habitat potentiel favorable au poisson, dans une portion de cours d'eau, en fonction de différents paramètres hydrodynamiques.

Elle est majoritairement utilisée dans 38 états d'Amérique du Nord (REISER et al., 1989) avec plus d'une centaine de cas d'application par an. L'expérience française repose sur une cinquantaine de cas conduits par le CEMAGREF (SOUCHON, 1994) ou par Electricité de France (SABATON et MIQUEL, 1993). Environ 500 cas de renouvellement légal de concessions hydroélectriques sont prévus dans les années futures, ce qui renforce le besoin de disposer d'une méthodologie correctement validée.

La relative jeunesse de la méthode des microhabitats, la nécessité de maîtriser plusieurs disciplines lors de son application, la complexité des différentes étapes et les multiples versions possibles ont provoqué des réactions de rejet et des critiques parfois vives dans la littérature (MATHUR et al., 1985 ; SHIRVELL, 1989 ; NEHRING et ANDERSON, 1993). L'interrogation majeure porte sur la signification biologique de la notion de capacité d'accueil potentielle et sur l'existence d'une relation, pour un débit limitant la population, entre la Surface Pondérée Utile calculée ("SPU", surface d'habitat favorable au poisson) et la biomasse de poissons observée.

En tant que développeurs et utilisateurs de la méthode des microhabitats depuis une dizaine d'années, il nous apparaît nécessaire de faire le point sur :

- la validité globale de la méthode c'est-à-dire jusqu'au niveau populationnel, en s'intéressant aux hypothèses fondatrices, aux conditions expérimentales à réunir pour les tester et aux résultats obtenus,

- la validation de chacune des étapes-clés : échantillonnage et simulation physiques, sensibilité aux différents types de modèles biologiques ; nous présenterons notamment l'intérêt de nouveaux modèles biologiques multivariés,

- les développements et efforts de recherche nécessaires pour en augmenter la fiabilité. Nous insisterons sur les développements récents que nous avons pu réaliser sur la partie biologique de la démarche. Enfin, nous discuterons d'une meilleure prise en compte de la dynamique temporelle et de la complexité de fonctionnement des rivières, ainsi que des perspectives d'application dans les grands cours d'eau.

\section{VALIDATION GLOBALE DE LA MÉTHODE}

L'espoir d'une validation globale jusqu'au niveau populationnel apparaît très vite comme un challenge fort avec des critiques initiales violentes de MATHUR et al. (1985) sur l'absence d'une démonstration évidente sur l'existence de corrélations entre la SPU et les caractéristiques d'une population. A cette époque, les premiers travaux fournissaient des conclusions encore mitigées selon les espèces et les types de milieux (ORTH et MAUGHAN, 1982 ; LOAR, 1985). Certains auteurs allant même jusqu'à dire qu'il est vain de rechercher ce type de relation (GORE et NESTLER, 1988). Compte tenu du temps de mise en place d'un réel retour d'expérience et de constitution d'un jeu de données significatif au plan populationnel, il est assez logique de ne voir émerger de réelles études de validation qu'après un laps de temps de l'ordre de la décennie (BOVEE, 1988 ; SOUCHON et al., 1989 ; JOWETT, 1992 ; NEHRING et ANDERSON, 1993). 
L'hypothèse simplificatrice initiale énoncée dans I'IFIM considère que l'effectif d'une population de poisson doit être directement corrélée avec la qualité de l'habitat. En réalité, certaines relations entre la phénologie des habitats et la dynamique des populations sont directes (ex. habitat de reproduction), alors que d'autres sont plus subtiles et complexes (ex. réussite de la phase d'incubation, puis réalisation du potentiel d'habitat estival pour expliquer le recrutement annuel d'une population).

Les conditions à réunir pour valider une éventuelle relation entre SPU et biomasse d'une population ont été énumérées par ORTH (1987) et BOVEE (1988) :

- la capacité d'accueil physique est saturée lors des mesures d'estimation du niveau de la population. Cette condition est difficile à obtenir puisque l'échantillonnage doit avoir lieu après des périodes de relative stabilité hydrologique (hors événement antérieur plus violent qu'une crue de fréquence de retour 2 ans considérée comme la crue morphogène, SEDELL et BESCHTA, 1991) dans les 3 ans précédant l'intervention. BOVEE (1988) signale qu'un déclin de la population adulte de truite est souvent constaté au bout de 2 à 3 ans de recrutement médiocre. L'échantillonnage ne doit pas être réalisé pendant les périodes où les poissons effectuent d'importantes migrations saisonnières (période de reproduction). Dans le cas de populations de salmonidés constituées simultanément de fractions sédentaires et migratrices, d'autres facteurs de contrôle que l'habitat sont susceptibles de jouer ;

- le rôle de l'habitat hydro-morphodynamique (hauteur, vitesse, substrat: H, V, S) est prépondérant sur les autres caractéristiques d'habitat (ex. végétation pour certains types de cours d'eau) sauf si l'on dispose de modèles biologiques à plus large spectre paramétrique. II constitue le facteur de contrôle ultime d'une population. II est considéré de façon dynamique au cours d'un cycle hydrologique. Une relation instantanée entre habitat et biomasse n'a a priori aucun sens, la population se structurant au cours d'épisodes-clés de son cycle vital. Apparaît ici la dimension temporelle très liée à l'hydrologie et la notion de période physique sensible ("bottleneck") pour une population, qui oblige à considérer aussi bien des durées d'événements que des intensités et des fréquences au moyen de techniques en cours de développement (CAPRA et al., 1995) ;

- les modèles biologiques retenus ont une validité régionale testée ;

- il n'existe pas d'interférence d'ordre physico-chimique ou thermique vis-à-vis de l'optimum de vie des espèces. Ce qui revient à n'étudier que des populations dans les conditions les plus naturelles existantes dans chaque région (stations de référence, NATIONAL RESEARCH COUNCIL, 1992). De même, les autres facteurs régulant une population, comme la trophie (ressources alimentaires) et l'équilibre dynamique général du cours d'eau ne doivent pas être altérés ;

- le stock ne doit pas être affecté par une pression de pêche trop forte.

En résumé, d'après BOVEE (1988), la prise en compte de l'habitat est une condition nécessaire mais pas suffisante pour expliquer le niveau auquel parvient une population. C'est pourquoi il faut aussi tenir compte des ressources nutritives, des relations de compétition, de prédation. Le même auteur conclut que "ce n'est pas parce que les relations entre habitat et populations de poissons s'avèrent complexes et difficiles à mettre en évidence qu'elles n'existent pas".

Toutes ces considérations expliquent pourquoi plusieurs études n'ont pu mettre en évidence des relations entre capacité d'habitat et biomasse d'une espèce, soit par nonrespect de conditions expérimentales, soit par existence de facteurs de contrôle ne dépendant pas directement de l'habitat, soit encore par application imparfaite des protocoles d'échantillonnage. En dehors des cas où la logique des microhabitats n'a pas été comprise, les absences de relations sont expliquées par :

- des espèces très ubiquistes pour l'utilisation de leur habitat (ORTH et MAUGHAN, 1982);

— des espèces aux exigences d'habitat encore mal décrites (NEHRING, 1979) ;

- une mauvaise prédiction physique locale pour une phase biologique très précise comme la reproduction (SHIRVELL, 1989). Dans ce cas, il est préférable d'effectuer une étude spécifique sur les frayères mêmes, en adaptant le pas de mesure à leur taille ;

- des populations en situation sympatrique, ce qui n'autorise plus l'utilisation de modèles biologiques bâtis sur des populations monospécifiques (LOAR, 1985). 
Les études qui concluent à l'existence d'une relation peuvent être classées en deux catégories suivant l'angle de recherche retenu:

- validations régionales : l'objectif est de tester régionalement sur le plus de cours d'eau possible l'existence d'une relation positive entre habitat estimé par la méthode des microhabitats et niveau des populations. II s'agit surtout d'examiner la validation générale de toute la démarche avec une hypothèse forte sur l'existence d'une période (saisonnière) limitante. SOUCHON et al. (1989) ont mis en évidence une relation linéaire entre la biomasse de truite fario adulte (longueur $>180 \mathrm{~mm}$ ) et la SPU de cette espèce calculée pour le débit minimum mensuel interannuel dans 12 cours d'eau de référence des Alpes, du Jura et du Massif Central (modules de 0,3 à 4,5 $\mathrm{m}^{3} \cdot \mathrm{s}^{-1}$ ). JOWETT (1992) a trouvé une relation significative entre SPU de truite fario adulte (longueur $>200 \mathrm{~mm}$ ) à l'étiage, SPU de production des invertébrés au débit médian et biomasse de truite adulte dans 59 cours d'eau de Nouvelle-Zélande (modules de 2 à $195 \mathrm{~m}^{3} . \mathrm{s}^{-1}$ ).

- validations temporelles : l'angle choisi ici consiste à suivre biologiquement quelques cours d'eau sur le moyen terme (de l'ordre d'une dizaine d'années) avec reconstitution des chroniques d'habitat pour les différents stades vitaux d'une espèce. Différentes relations sont alors recherchées stade par stade, vis-à-vis de leurs contraintes d'habitat respectives. BOVEE (1988) puis NEHRING et ANDERSON (1993) ont ainsi mis en évidence le rôle déterminant des conditions d'habitat pendant et peu après l'émergence (jusqu'à 2 à 4 semaines) pour 11 cours d'eau du Colorado (modules de 3,5 à $40 \mathrm{~m}^{3} \cdot \mathrm{s}^{-1}$ ). Ils démontrent ainsi que pour ces populations d'altitude (1660 $\mathrm{m}$ à $2800 \mathrm{~m}$ ), I'habitat est fortement contraint par l'hydrologie particulière de chaque année. CAPRA et al. (1995) proposent une méthode de calcul des valeurs hydrologiques seuils assorties de durées biologiquement significatives pour mieux prendre en compte cette dynamique temporelle d'habitat.

\section{VALIDATION DE CHACUNE DES ÉTAPES}

\section{Modèles hydrauliques}

Les modèles hydrauliques n'ont pas fait l'objet d'une grande critique dans les exemples d'application de la méthode des microhabitats. Pour le modèle IFG4 utilisé dans le module américain de simulation de l'habitat (PHABSIM : BOVEE et MILHOUS, 1978), NERHING (1979) indique des erreurs de $10 \%$ pour $35 \%$ des mesures, de plus de $100 \%$ pour $4 \%$ des mesures. Le modèle Fluvia (BAUME et POIRSON, 1984) modifié avec l'équation de Limerinos (TROCHERIE, 1987), est utilisé dans le logiciel EVHA (GINOT et SOUCHON, 1995). II conduit à afficher un taux d'erreur de reconstitution de l'ordre de $10 \%$ des valeurs. Cependant ces chiffres sont sous-estimés dans le cas d'application de ces modèles dans des cours d'eau à géométries complexes et avec de faibles tirants d'eau par rapport à la taille du substrat (MALAVOI et al., 1989). De nouvelles recherches sont actuellement en cours pour la mise au point de modèles plus performants dans des milieux plus torrentiels (HEROUIN et al., 1995).

\section{Modèles biologiques}

Le type de modèle biologique utilisé lors du couplage conditionne grandement l'estimation des valeurs d'habitat et leur signification. Les modèles biologiques les plus largement répandus pour l'application de la méthode des microhabitats se présentent sous la forme de profils écologiques monovariés établis pour différents paramètres physiques. Les exigences des stades de développement d'une espèce pour les 3 paramètres physiques (Hauteur, Vitesse, Substrat : $H, V, S$ ), considérés comme prépondérants dans la sélection des habitats par les poissons, sont exprimées sous la forme de fonctions de préférence (codées de 0 à 1). Ces courbes sont établies soit par synthèse bibliographique, soit par enquêtes auprès d'experts ou encore à partir de mesures directes (BOVEE, 1986).

Ce type de courbes de préférence fait l'objet de deux critiques principales (MATHUR et al., 1985 ; ORTH, 1987 ; SHIRVELL, 1989):

- la non prise en compte de la dépendance des variables hydro-morphodynamiques. Pour justifier leur démarche, BOVEE et MILHOUS (1978) ont avancé que les poissons 
sélectionnaient de façon indépendante les différents paramètres physiques de leur habitat. Plusieurs études ont pu montrer que cette hypothèse ne se vérifiait pas toujours, notamment entre la hauteur d'eau et la vitesse du courant (ORTH et MAUGHAN, 1982). Pour lever cette difficulté, certains auteurs ont alors proposé des courbes bivariées (LAMBERT et HANSON, 1989), peu appliquées à notre connaissance ;

- la nécessité de prise en compte d'autres variables, les seuls paramètres H,V,S n'étant pas suffisants pour expliquer la répartition des poissons. L'importance des abris a par exemple été soulignée par de nombreuses études (FAUSCH et al., 1988), et d'autres remarques ont eu trait à l'influence des relations biotiques intra ou interspécifiques.

La sélection de l'habitat par les poissons s'effectue donc en fonction d'un ensemble de conditions biotiques et abiotiques, plutôt que par la juxtaposition d'exigences monovariées. II était donc légitime de transcrire ces exigences par des modèles multivariés. Si de nombreux auteurs ont affirmé cette nécessité, peu l'ont concrétisée. De récents développements dans ce domaine nous ont permis de comparer la qualité prédictive de courbes de préférence et de modèles multivariés. Ces modèles correspondent à des analyses discriminantes réalisées à partir des données préalablement transformées par analyse multivariée (POUILLY et SOUCHON, 1994). L'espace factoriel issu de cette analyse conduit à une typologie des habitats, établie en fonction de leurs caractéristiques physiques et de leur composition biologique. L'analyse discriminante détermine les sous-espaces factoriels composés d'habitat où l'espèce est présente et ceux où elle est absente. Après projection d'un nouvel habitat dans l'espace factoriel, la fonction discriminante définit s'il se situe dans un sous-espace favorable ou défavorable à la présence de l'espèce. Ces modèles multivariés ont été testés sur trois espèces de cyprinidés (barbeau: Barbus barbus ; chevaine : Leusciscus cephalus ; gardon : Rutilus rutilus). Les résultats ont notamment établi que les modèles multivariés ont une qualité de prédiction supérieure (de l'ordre de $10 \%$ ) à celle des courbes de préférence (POUILLY, 1994). De plus, pour chaque type de modèle, une comparaison a été réalisée entre des modèles locaux (spécifiques d'un site) et des modèles généraux (applicables sur un ensemble de sites). Les courbes de préférence monovariées n'ont pas le caractère généralisable attendu, il est donc préférable de recourir à des modèles locaux. A l'inverse, les modèles multivariés généraux gardent une bonne qualité de prédiction (POUILLY et SOUCHON, 1994). Cette conclusion est essentielle pour autoriser l'utilisation des modèles sur l'ensemble des cours d'eau d'une même région et donc pour faciliter l'utilisation de la méthode des microhabitats.

\section{DISCUSSION ET PERSPECTIVES}

La méthode des microhabitats apparaît complexe en raison des compétences disciplinaires à mobiliser. C'est à l'évidence un outil qu'il faut utiliser de façon dynamique avec un bon niveau de connaissance sur l'écologie et le fonctionnement des cours d'eau. Sa validité est désormais suffisamment démontrée pour en faire un des outils précieux d'analyse prévisionnelle de l'impact des altérations physiques sur I'habitat aquatique et sur la dynamique biologique associée. Son évolution future devra incorporer différents résultats de recherche dans chacun de ses domaines physiques et biologiques, mais aussi s'intégrer dans une panoplie plus large d'outils d'analyse du fonctionnement général des hydrosystèmes lotiques. Ces outils devront concerner par exemple l'appréciation de l'équilibre dynamique des cours d'eau. C'est pourquoi la réelle nécessité de valider la démarche par confrontation avec la biomasse d'une population ne doit pas conduire à l'enfermer dans la catégorie des méthodes de prédiction des biomasses de poissons.

Plusieurs progrès sont attendus, dont l'opportunité de mise en oeuvre sera à juger en fonction des cas à traiter et de leurs enjeux :

- étude de la dynamique hydrologique et de l'utilisation spatio-temporelle de l'habitat des poissons. A l'échelle annuelle, deux types d'habitats peuvent être distingués en fonction de leur fréquence d'utilisation par les poissons : des habitats "exceptionnels" et des habitats "quotidiens". Les premiers sont utilisés sur de courtes périodes de temps pendant lesquelles le comportement des poissons est modifié, soit par des événements hydrologiques extrêmes (crue ou étiage), soit par la reproduction. Les mouvements des poissons sont alors amplifiés et dépassent le cadre spatial habituel, qualifiable de domaine 
vital "quotidien". D'où une signification différente des capacités d'accueil, pour une même valeur de SPU, à faible débit et à fort débit. Des améliorations sont à attendre des travaux en cours sur l'analyse des séries temporelles d'habitat en termes de seuils, de durée et de fréquence (méthode Durée d'Habitat Limitant DHL : CAPRA et al., 1995) ;

- extension de la méthode aux grands cours d'eau. STALNAKER et al. (1989) et POUILLY (1994) n'y voient pas de difficultés majeures notamment au niveau de la modélisation physique. Toutefois, il est nécessaire d'obtenir une meilleure prise en compte de la complexité morphodynamique de ces cours d'eau. Elle dépend de la capacité de résolution des modèles hydrauliques sachant que les systèmes en tresse sont encore mal traités et que les bras latéraux sont peu pris en compte, contrairement au chenal principal. Pourtant ces annexes sont des habitats essentiels pour la dynamique des peuplements de poissons. De nouvelles approches de modélisation des conditions hydrodynamiques nécessitant moins de mesures de terrain voient le jour (LAMOUROUX et al., 1992 ; LE COARER et DUMONT, 1995) et aideront certainement à résoudre ces points.

Au niveau biologique, certains travaux (ANGERMEIER, 1987 ; ROSS et al., 1987) concluent que les poissons des zones potamiques ont des exigences moins strictes vis-àvis de l'habitat que les espèces du rhitron. Des travaux récents sur les cyprinidés rhéophiles montrent que si l'utilisation de l'habitat est complexe, il existe bien des relations entre I'habitat et les poissons (BARAS et CHERRY, 1990 ; POUILLY, 1994). La multiplicité des espèces, des stades de développement et des facteurs influençant le choix du microhabitat limite l'utilisation de courbes de préférence monovariées : la multiplication du nombre de courbes ne peut à elle seule traduire cette complexité. II est alors nécessaire de développer des nouveaux types d'approche permettant une traduction multivariée de l'information (statistique multivariée, réseaux de neurones).

Dans ces systèmes, le nombre d'espèces correctement décrites pour leurs exigences d'habitat est encore insuffisant. Pour rendre compte de toute la complexité du vivant, il faut intégrer de plus en plus d'espèces de poissons en ne négligeant pas les espèces rares. Une réelle stratégie doit être mise en place pour choisir entre des approches privilégiant des espèces et stades cibles, des guildes d'exigences d'habitat (LEONARD et ORTH, 1988) ou tout un peuplement de poissons. Cette dernière voie est certainement à encourager puisque les modèles multivariés l'autorisent désormais (POUILLY, 1994).

A notre sens, la partie biologique de la démarche microhabitat doit encore être étoffée aussi bien au niveau des modèles biologiques d'exigences d'habitat selon les axes proposés ci-avant qu'au niveau de la dynamique des populations et des peuplements. Les premières tentatives allant dans cette dernière voie (JAGER et al., 1993) en montrent la difficulté, notamment par la nécessité de disposer de séries biologiques sur le long terme. Cependant tout l'intérêt de ces démarches sera de pouvoir réellement mettre en évidence les phases spatio-temporelles sensibles pour la dynamique des communautés, qui représentent les leviers sur lesquels il est possible d'agir pour restaurer le fonctionnement biologique des cours d'eau anthropisés.

\section{BIBLIOGRAPHIE}

ANGERMEIER P.L., 1987. Spatiotemporal variation in habitat selection by fishes in small Illinois streams in Matthews W.J., Heins D.C., Community and Evolutionary Ecology of North American Stream Fishes, University of Oklahoma Press, Norman : 52-60.

BARAS E., CHERRY B., 1990. Seasonal activities of female barbel (Barbus barbus, L.) in the river Ourthe as revealed by radio tracking. Aquat. Living Resour., 3 (4), 283-294.

BAUME J.P., POIRSON M., 1984. Modélisation numérique d'un écoulement permanent dans un réseau hydraulique maillé à surface libre, en régime fluvial. La Houille Blanche, 1 (2), 95-100.

BOVEE K.D., 1982. A guide to stream habitat analysis using the Instream Flow Incremental Methodology. Instream Flow Information Paper $n^{\circ} 12$, FWS/OBS 82/86, 248 p. 
BOVEE K.D., 1986. Development and evaluation of habitat suitability criteria for use in the instream flow incremental methodology. Instream Flow Information Paper $n^{\circ} 21$, FWS/OBS 86/7, $188 \mathrm{p}$.

BOVEE K.D., 1988. Use of the instream flow incremental methodology to evaluate influences of microhabitat variability on trout populations in four Colorado streams. 68th Annual conference, Western Association of Fish and Wildlife Agencies, Albuquerque, $31 \mathrm{p}$.

BOVEE K.D., MILHOUS R.T., 1978. Hydraulic simulation in instream flow studies: theory and techniques. Instream Flow Information Paper $n^{\circ}$ 5, FWS/OBS 78/33, $130 \mathrm{p}$.

CAPRA H., BREIL P., SOUCHON Y., 1995. A new tool to interpret magnitude and duration of fish habitat variations. Reg. Riv. : Res. and Manag., in press.

FAUSCH K.D., HAWKES C.L., PARSONS M.G., 1988. Models that predict standing crop of stream fish from habitat variables: 1950-85. General technical report, U.S. Agriculture, PNW-GTR-213.

GINOT V., SOUCHON Y., 1995. Logiciel EVHA. Evaluation de I'habitat physique des poissons en rivière (version 1.0, beta test). Vol. 1. Guide méthodologique, $106 \mathrm{p}$. Vol. 2. Guide de l'utilisateur, $66 \mathrm{p}$. Cemagref BEA/LHQ Lyon, France, et Ministère de l'Environnement, Dir. de l'Eau, Paris, France.

GORE J.A., NESTLER J.M., 1988. Instream flow studies in perspective. Reg. Riv. : Res. and Manag., 2, 93-101.

HEROUIN E., BREIL P., ALBERT M.B., 1995. Quelles approches pour synthétiser le fonctionnement physique des écosystèmes d'eaux courantes? In : Symposium "Relations entre le poisson et son habitat", Lyon, 6-8 Décembre 1994. Bull. Fr. Pêche Piscic., 337/338/339.

JAGER H.I., DEANGELIS D.L., SALE M.J., WINKLE W.V., SCHMOYER D.D., SABO M.J., ORTH D.J., LUKAS J.A., 1993. An individual-based model for smallmouth bass reproduction and young-of-year dynamics in streams. Rivers, 4 (2), 91-113.

JOWETT I.G., 1992. Models of the abundance of large brown trout in New Zealand rivers. N. Am. Fish. Manag., 12, 417-432.

LAMBERT T.R., HANSON D.F., 1989. Development of habitat suitability criteria for trout in small streams. Reg. Riv. : Res. and Manag., 3, 291-303.

LAMOUROUX N., STATZNER B., FUCHS U., KOHMANN F., SCHMEDTJE U., 1992. An unconventional approach to modelling spatial and temporal variability of local shear stress in stream segments. Water Res., 28 (12), 3251-3258.

LE COARER Y., DUMONT B., 1995. Modélisation de la morphodynamique fluviale pour la recherche des relations habitat-faune aquatique. In: Symposium "Relations entre le poisson et son habitat", Lyon, 6-8 Décembre 1994. Bull. Fr. Pêche Piscic., 337/338/339.

LEONARD P.M., ORTH D.J., 1988. Use of habitat guilds of fishes to determine Instream Flow requirements. N. Am. Fish. Manag., 8, 399-409.

LOAR J.M., 1985. Application of habitat evaluation models in Southern Appalachian trout streams. Environmental sciences division, $310 \mathrm{p}$.

MALAVOI J.R., SOUCHON Y., TROCHERIE F., 1989. Etude de l'Arc à Modane (Savoie). Application de la méthode des microhabitats, aspects méthodologiques. CEMAGREF / DPN, convention 88/321, $25 \mathrm{p}$.

MATHUR D., BASON W.H., PURDY E.J., SILVER C.A., 1985. A critique of the Instream Flow Incremental Methodology. Can. J. Fish. Aqua. Sci., 42, 825-831.

NATIONAL RESEARCH COUNCIL (U.S.), 1992. Restoration of aquatic ecosystems : science, technology, and public policy. National Academy Press, Washington D.C., 552 p. 
NEHRING R.B., 1979. Evaluation of instream flow methods and determination of water quantity needs for streams in the state of Colorado. Fort Collins, Division of Wildlife, $\mathrm{n}^{\circ}$ 14-16-00006-78-909, $144 \mathrm{p}$.

NEHRING R.B., ANDERSON R.M., 1993. Determination of population limiting critical salmonid habitats in Colorado streams using the physical habitat simulation system. Rivers, 4 (1), 1-19.

ORTH D.J., 1987. Ecological considerations in the development and application of instream flow habitat models. Reg. Riv. : Res. and Manag., 1, 171-181.

ORTH D.J., MAUGHAN O.E., 1982. Evaluation of the incremental methodology for recommending instream flow for fishes. Trans. Am. Fish. Soc., 3 (4), 413-445.

POUILLY M., 1994. Relations entre l'habitat physique et les poissons des zones à cyprinidés rhéophiles dans trois cours d'eau du bassin rhodanien : vers une simulation de la capacité d'accueil pour les peuplements. Thèse Univ. Lyon I/ CEMAGREF Lyon, $260 \mathrm{p}$.

POUILLY M., SOUCHON Y., 1994. Simulation de l'habitat physique du barbeau fluviatile (Barbus barbus, L.1758) : choix des modèles biologiques et sensibilité de la réponse. Bull. Fr. Pêche Piscic., 334, 213-225.

REISER D.W., WESCHE T.A., ESTES C., 1989. Status of instream flow legislation and practices in North America. Fisheries, 14(2), 22-29.

ROSS S.T., BAKER J.A., CLARK K.E., 1987. Microhabitat partitioning of southeastern stream fishes : temporal and spatial predictability in Matthews W.J., Heins D.C., Community and Evolutionary Ecology of North American Stream Fishes, University of Oklahoma Press, Norman : 42-51.

SABATON C., MIQUEL J., 1993. La méthode des microhabitats : un outil d'aide au choix d'un débit réservé à l'aval des ouvrages hydroélectriques. Expérience d'Electricité de France. Hydroécologie Appliquée, 5 (1), 127-163.

SEDELL J.R., BESCHTA R.L., 1991. Bringing back the "Bio" in bioengineering. Am. Fish. Soc. Symp., 10,160-175.

SHIRVELL C.S., 1989. Ability of Phabsim to predict chinook salmon spawning habitat. Reg. Riv. : Res. and Manag., 3, 277-289.

SOUCHON Y., 1994. Etat d'avancement des recherches sur la modélisation de I'habitat des poissons en France. Bull. Fr. Pêche Piscic., 332, 57-71.

SOUCHON Y., TROCHERIE F., FRAGNOUD E., LACOMBE C., 1989. Les modèles numériques des microhabitats des poissons : application et nouveaux développements. Rev. Sci. Eau, 2, 807-830.

STALNAKER C.B., MILHOUS R.T., BOVEE K.D., 1989. Hydrology and hydraulics applied to fishery management in large rivers. Can. Spec. Publ. Fish. Aqua. Sci., 106, 13-30.

TROCHERIE F., 1987. Mise en application de l'article 410 du Code Rural. Elément d'une démarche de détermination des débits réservés. CEMAGREF / DPN, 85-15, 30 p. 